\title{
SAN099.2151C
}

\section{EDGE STATE PROPAGATION DIRECTION IN THE FRACTIONAL \\ QUANTUM HALL REGIME: MULTI-TERMINAL MAGNETOCAPACITANCE EXPERIMENT.}

\author{
J. S. Moon ${ }^{a}$, J. A. Simmons ${ }^{a}$, B. L. Johnson ${ }^{b}$ J. L. Reno \\ ${ }^{a}$ Sandia National Laboratories, Albuquerque, NM 87185 USA \\ ${ }^{b}$ Department of Physics, Western Washington University, Bellingham, Washington 98225 USA
}

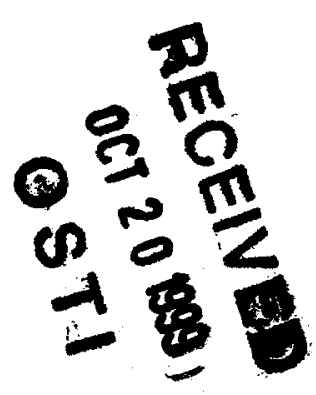

\section{Corresponding Author:}

J. S. Moon, Mail Stop 1415, Sandia National Laboratories, P. O. Box 5800, Albuquerque, NM 87185-1415, USA

Fax: (505) 844-1197; E-mail: jmoon@sandia.gov

The propagation direction of fractional quantum Hall effect (FQHE) edge states has been investigated experimentally via the symmetry properties of the multi-terminal capacitances of a two dimensional electron gas. Although strong asymmetries with respect to zero magnetic field appear, no asymmetries with respect to even denominator Landau level filling factor $v$ are seen. This indicates that current-carrying FQHE edge states propagate in the same direction as integer QHE edge states. In addition, anomalous capacitance features, indicative of enhanced bulk conduction, are observed at $v=1 / 2$ and $3 / 2$.

PACS numbers: 73.40.Hm, 71.10.Pm, 73.20.Dx, 73.50.Jt

Keywords: edge states, capacitance, integer and fractional quantum Hall effect. 


\section{DISCLAIMER}

This report was prepared as an account of work sponsored by an agency of the United States Government. Neither the United States Government nor any agency thereof, nor any of their employees, make any warranty, express or implied, or assumes any legal liability or responsibility for the accuracy, completeness, or usefulness of any information, apparatus, product, or process disclosed, or represents that its use would not infringe privately owned rights. Reference herein to any specific commercial product, process, or service by trade name, trademark, manufacturer, or otherwise does not necessarily constitute or imply its endorsement, recommendation, or favoring by the United States Government or any agency thereof. The views and opinions of authors expressed herein do not necessarily state or reflect those of the United States Government or any agency thereof. 


\section{DISCLAIMER}

Portions of this document may be illegible in electronic image products. Images are produced from the best available original document. 
For a two dimensional electron gas (2DEG) in the integer quantum Hall effect (IQHE) regime, the concept of edge states provides a unified description of transport phenomena. [1] A number of experiments such as capacitance measurements [2-4], inductive probing [5], and local imaging techniques [6] have been used to investigate edge state phenomena in the IQHE regime.

In the fractional quantum Hall effect (FQHE) regime where the single particle picture is no longer valid, the composite fermion (CF) [7] approach has been strongly supported by experimental results, including measurements of the FQHE energy gaps [8] and Fermi surface effects at even denominator Landau level filling factor $v$, where the effective magnetic field $\mathrm{B}_{\text {eff }}=0 .[9,10]$ Recently, the $\mathrm{CF}$ approach has been extended to edge states in the FQHE regime [11, 12], which are all expected to propagate in the same direction as for the IQHE. To date few experiments have addressed the propagation direction of edge states. Ashoori et al., studied the propagation direction of edge magnetoplasmons and found the same propagation direction for $v=1$ and $v=2 / 3$. [13] However, the relevance of this result to the propagation direction of $\mathrm{FQHE}$ edge states per se is unclear. Later, Chen et al. [3] used a magnetocapacitance measurement with multiple $2 \mathrm{DEG}$ contacts to determine the propagation direction of IQHE edge states.

In this paper, we extend the multiterminal magnetocapacitance technique of Chen et al. to a high mobility $2 \mathrm{DEG}$ to study the propagation direction of the edge states in the integer and fractional QHE regime. [4] Unlike conventionàl two-terminal capacitance measurements, here the capacitance is measured between a single gate and 3 separated, grounded ohmic contacts to the 2DEG. (See inset to Fig. 1.) Thus, in this multi-terminal configuration, capacitive coupling to each ohmic contact is strongly influenced by the propagation direction of the current carrying channels, rather than simply the total 
density of states (DOS). The resulting capacitance becomes a tensor quantity $\mathrm{C}_{\mathrm{G}, \mathrm{k}}(\omega)$, given by the current $I_{k}(\omega)$ measured at contact $k$, divided by the voltage modulation at the gate $V(\omega)$. The edge states are presumed to follow the edge of the sample and to terminate at the contacts $\mathrm{k}$. Therefore $\mathrm{C}_{\mathrm{G}, 1}$ and $\mathrm{C}_{\mathrm{G}, 3}$ are sensitive to the edge state direction, while $\mathrm{C}_{\mathrm{G}, 2}$ becomes significant only when the bulk is compressible and current can flow through it. By studying the symmetry properties of the magnetocapacitance tensor, we found that (1) for the soft-wall boundaries of our sample, the current-carrying FQHE edge states on either side of $3 / 2$ and $1 / 2$ all propagate in the same direction as the IQHE edge states. (2) Near $v=1 / 2$ and $3 / 2\left(B_{\text {eff }} \approx 0\right)$ the average motion of CFs is also strongly chiral with the sign of chirality determined by $B$ rather than $B_{\text {eff. }}$ These observations are consistent with recent theoretical predictions. [11, 12] (3) Finally, we observe anomalous broad flat-topped peaks at filling factors $v=1 / 2$ and $3 / 2$, in the capacitance between the gate and a $2 D E G$ contact directly connected to it only by paths through the bulk.

Characteristics of the three high-mobility GaAs/AlGaAs heterostructures investigated are listed in Table 1. The sample geometry is shown schematically in the inset to Fig. 1. An AC modulation voltage $\mathrm{Ve} \mathrm{e}^{\mathrm{i} \omega t}$ (typically $1 \mathrm{mV}$ ) is applied to gate $\mathrm{G}$, a semitransparent metallic surface gate composed of $\sim 300 \AA$ of $\mathrm{Cr}$, which covers the edge of the 2DEG mesa and much of the bulk. The out-of-phase current $I_{k}(\omega)$ (of order $10 \mathrm{pA}$ ) is then simultaneously measured at the three diffused metallic $2 \mathrm{DEG}$ contacts $\mathrm{k}=1,2,3$, using phase-sensitive current-preamplifiers with impedance to ground $\leq 5 \Omega$. The capacitance tensor elements $C_{G, k}$ are directly given by $I_{k}(\omega) /(\omega V(\omega))$. The in-phase 
current is negligible with sufficiently low $\omega$ (typically $200 \mathrm{~Hz}$ ). The gate and contact dimensions and separations were typically of order $1 \mathrm{~mm}$.

Fig. 1(a)-(c) shows $C_{G, k}$ of sample $E A 100$ for $T=0.3 \mathrm{~K}$. (The $C_{G, k}$ 's were measured simultaneously, so all 2DEG contacts were nominally at ground potential.) Fig. 1(d) shows $\rho_{x x}$, along with the two terminal capacitance $C_{2 T}$, obtained by allowing two of the 2DEG contacts to float. The two-terminal capacitance shows dips at the quantum Hall plateau, reflecting the incompressible nature of the 2DEG bulk regions. The reversal of B field doesn't change the magnitude of the two-terminal capacitance, which remains completely symmetric with $\mathrm{B}$. In contrast, both the 'edge-connected' capacitances $\mathrm{C}_{\mathrm{G}, 1}$ and $\mathrm{C}_{\mathrm{G}, 3}$ exhibit a striking asymmetry upon reversal of $\mathrm{B}$. For positive $\mathrm{B}, \mathrm{C}_{\mathrm{G}, 1}$ exhibits a large background with superimposed dips associated with the formation of incompressible states in the bulk. At negative $\mathrm{B}$ however, $\mathrm{C}_{\mathrm{G}, 1}$ drops nearly to zero. $\mathrm{C}_{\mathrm{G}, 3}$ exhibits similar behavior, only in reverse. The 'bulk-connected' capacitance $\mathrm{C}_{\mathrm{G}, 2}$, on the other hand, exhibits a large peak at $B=0$, but falls off rapidly and symmetrically as $B$ deviates from zero. Qualitatively similar results are obtained for the other samples.

Our results in the IQHE regime are conceptually simplest to understand when $v=$ integer. We use the conventional edge state picture, where the bulk is incompressible $\left(\sigma_{x x}=0\right)$ and an induced current is carried only in the edge channels. For positive B, when the gate voltage changes, current injected from contact 3 -the 'edge-state sourcing contact'- remains unchanged, since it is determined only by the chemical potential in the ungated regions. (See Fig. 1 inset.) However, current flowing into contact 1 -the 'edgestate sinking contact'-is changed, since charge is accumulating under the gate. This results in a net, non-zero current appearing at contact 1, proportional to the area of the edge states. When $B$ is negative, the roles of contacts 1 and 3 are interchanged as the 
edge-state propagation direction reverses. This results in the observed asymmetry between $\mathrm{C}_{\mathrm{G}, 1}$ and $\mathrm{C}_{\mathrm{G}, 3}$. On the other hand, $\mathrm{C}_{\mathrm{G}, 2}$, symmetric with respect to $\mathrm{B}=0 \mathrm{~T}$, drops towards zero for $v=$ integer, since it is connected to the gate only by incompressible states in the bulk.

Outside of the quantum Hall plateaus where the bulk is compressible, the asymmetry is also present except for a slight residual capacitance. Indeed, the asymmetry is almost completely developed at $\mathrm{B}= \pm 0.05 \mathrm{~T}$, even at high temperatures. $[3,4]$ This indicates that the asymmetry is classical in origin, appearing when B is large enough to change the transport from an isotropically diffusive regime to one in which the Lorentz force is relevant. We have developed a quantitative model, described elsewhere [4], based on the bulk transmission probabilities between various contacts in the ungated region, where the capacitive coupling is determined by both $\sigma_{\mathrm{xx}}$ and $\sigma_{\mathrm{xy}}$. Excellent agreement with the data was found.

We now discuss our data in the $\mathrm{FQHE}$ regime. In the $\mathrm{CF}$ picture, the $\mathrm{FQHE}$ is viewed as the IQHE of CFs in the presence of an effective magnetic field $B_{\text {eff }}=B-B_{g}$, where $B_{g}=2 m n h / e, m=$ integer and $n$ the $2 D E G$ density. At $m=1$ for instance, $B_{\text {eff }}$ is zero at $v=1 / 2$, positive for $v<1 / 2$ and negative for $v>1 / 2$. Geometric resonance experiments [10] have shown that CFs move semiclassically in the presence of small $B_{\text {eff }}$, similar to electrons near $B=0 \mathrm{~T}$. [14] Naively, one would then expect the appearance of asymmetrical features in $\mathrm{C}_{\mathrm{G}, \mathrm{k}}$ near $\mathrm{B}_{\text {eff }}=0$, analogous to those seen near $\mathrm{B}=0$, and indicating a reversal in the FQHE edge state propagation direction. However, as is apparent in Fig. 1, at $v=1 / 2$ no sudden transition nor clear signature of an asymmetry is present in $C_{G, 1}$ nor $C_{G, 3}$. While nearby features associated with the fractions $4 / 3$ and $5 / 3$ 
make the situation less clear, at $v=3 / 2$ no clear asymmetry is apparent either. All three samples behaved similarly.

To verify the absence of any asymmetries with respect to $B_{\text {eff }}=0$, the gate excitation voltage was varied from $0.5 \mathrm{mV}$ to $5 \mathrm{mV}$ and no change in the capacitive signal was found. Assuming an effective CF mass of $\sim 0.67 \mathrm{~m}_{e}$, [8] the quasiparticle energy gap at $v$ $=1 / 3(\mathrm{~B}=16.2 \mathrm{~T})$ is estimated as $0.93 \mathrm{meV}$, much larger than the gate-induced Fermi energy modulation of a few $\mu \mathrm{eV}$. Further, the frequency was varied from $10 \mathrm{~Hz}$ to several $\mathrm{kHz}$ and no significant quadrature component due to a finite $\sigma_{\mathrm{xx}}$ was observed.

We also studied the gate-induced reflection of individual FQHE edge states from the same wafers, using a four-terminal configuration. [17] As shown in Fig. 2, several fractionally quantized plateaus in $R_{x x}$ are clearly observed. The quantized values of $R_{x x}$ agree well with the Landauer-Büttiker formalism [16], $R_{x x}=\left(1 / v_{g}-1 / v_{b}\right)\left(h / e^{2}\right)$, where $v_{\mathrm{b}}$ and $v_{\mathrm{g}}$ are the filling factors in the bulk and beneath the gate, respectively. Our results clearly indicate well-defined FQHE edge states in these wafers.

The observed lack of magneto-capacitance asymmetries in the FQHE indicates strongly that the current carrying FQHE edge states all propagate in the same direction as electrons in IQHE edge states, determined by $\mathrm{B}$ rather than $\mathrm{B}_{\text {eff }}$. This is consistent with recent theories [11], which indicate that the average (drift) motion of CFs is associated with the drift of the attached magnetic flux, and hence generates a fictitious gauge electric field $\mathrm{E}_{\mathrm{g}}=-(\mathrm{J} \cdot \hat{\mathrm{B}}) \mathrm{mh} / \mathrm{e}^{2}, \mathrm{E}_{\mathrm{g}}$ exerts a force $\mathrm{e}\langle\mathrm{v}\rangle \times \mathrm{B}_{\mathrm{g}}$, which exactly cancels the average force due to the fictitious magnetic field. As a result, the average force seen by CFs is the same as for electrons, yielding a CF edge state propagation direction which is 
the same as for electrons. In the ballistic regime, the individual $\mathrm{CF}$ moves at the Fermi velocity and the average drift force $e\langle v\rangle \times B_{g}$ has negligible effect on the semiclassical motion of CFs near $B_{\text {eff }}$, consistent with experiments [10].

We now turn to the anomalous capacitance peaks. A striking feature of the CF theory is the implication that at $\mathrm{B}_{\mathrm{eff}}=0$ there exists a Fermi surface with a well-defined Fermi wavevector $\mathrm{k}_{\mathrm{F}}=(4 \pi \mathrm{n})^{1 / 2}$. Since the measured $\mathrm{C}_{\mathrm{G}, \mathrm{k}}$ depend strongly on the bulk transport properties of the ungated regions, one might expect that they would exhibit a CF Fermi surface signature near $B_{\text {eff }}=0$. In Fig. 3(a) we show $C_{G, 2}$ for sample EMC715 at $0.3 \mathrm{~K}$. A distinct broad flat-topped peak, centered about $v=3 / 2$, is apparent. The peak has a sharp turn-on at $B-B_{3 / 2}=\Delta B_{3 / 2} \approx \pm 0.26 T$, yielding a normalized half-width of $\Delta B_{3 / 2} / B_{3 / 2}$ $\approx 0.036$. A smaller 'copy' of the peak [Fig. 3(b)] is apparent in $\mathrm{C}_{\mathrm{G}, 3}$, while an inverted peak (or broad flat-bottomed dip) is visible in $\mathrm{C}_{\mathrm{G}, 1}$. The inverted peak in $\mathrm{C}_{\mathrm{G}, 1}$ is expected from current conservation: an increase in bulk current must be compensated by a decrease in current along the edge. As temperature $T$ is increased [Fig. 3(c)], the peak becomes smaller and rounded, nearly disappearing by $1.2 \mathrm{~K}$. By contrast, $\rho_{\mathrm{xx}}$ shows no sharp features around $3 / 2$, exhibiting instead a gentle, temperature insensitive minimum. (Similar features, described elsewhere [4], are observed in $\mathrm{C}_{\mathrm{G}, 2}$ for sample EA100 and EA65 near $v=1 / 2$ ).

These anomalous features at even denominator $v$ cannot be attributed to changes in the DOS of the gated $2 D E G$ region, since in that case a peak near $3 / 2$ or $1 / 2$ would appear for $\mathrm{C}_{2 \mathrm{~T}}$ and for all $\mathrm{C}_{\mathrm{G}, \mathrm{k}}$, contrary to what we observe. Rather, we speculate that the anomalous capacitance features are due to an enhancement in the bulk conductivity near 
$\mathrm{B}_{\text {eff }}=0$ outside the gate, which, as we have seen, can strongly influence the multiterminal capacitance. We note that the capacitance features' characteristic temperature of order $1 \mathrm{~K}$ is similar to that predicted for the flux-binding energy of CFs. [18]

Work supported by the U.S. Department of Energy under Contract DE-AC04-

94AL85000.

Sandia is a multiprogram laboratory operated by Sandia Corporation, a Lockheed Martin Company, for the United States Department of Energy under contract DE-AC04-94AL85000. 
References

[1] B. I. Halperin, Phys. Rev. B 2 5, 2185 (1982).

[2] S. Takaoka et al., Phys. Rev. Lett. 72, 3080 (1994).

[3] W. Chen, T. P. Smith III, M. Büttiker, and M Shayegan, Phys. Rev. Lett. 73, 146 (1994). See also M. Büttiker, J. Phys. C 5, 9361 (1993).

[4] J. S. Moon et al., Phys. Rev. Lett. 79, 4457 (1997)

[5] E. Yahel et al., Phys. Rev. Lett. 81, 5201 (1998).

[6] S. H. Tessmer et al., Nature (London) 392, 51 (1998);K. L. McCormick et al., Phys. Rev. B 59, 4654 (1999).

[7] J. K. Jain, Phys. Rev. Lett. 63, 199 (1989); B. I. Halperin, P. A. Lee, and N. Read, Phys. Rev. B 47, 7312 (1993).

[8] R. R. Du et al., Phys. Rev. Lett. 70, 2944 (1993).

[9] R. L. Willet, R. R. Ruel, K. W. West, and L. N. Pfeiffer, Phys. Rev. Lett. 71, 3846 (1993).

[10] W. Kang, H. L. Stormer, and L. N. Pfeiffer, Phys. Rev. Lett. 7 1, 3850 (1993); V. J. Goldman, B. Su, and J. K. Jain, ibid. 72, 2065 (1994); J. H. Smet et al., ibid. 77, $2272(1996)$.

[11] G. Kirczenow and B. L. Johnson, Phys. Rev. B 51, 17579 (1995); G. Kirczenow, Phys. Rev. B 58, 1457 (1998);

[12] H.-S. Kim, K. J. Chang, and G. Ihm, Phys. Rev. Lett. 82,596 (1999).

[13] R. C. Ashoori et al., Phys. Rev. B 4 5, 3894 (1992).

[14] H. van Houten et. al., Phys. Rev. B 39, 8556 (1989); J. S. Moon et. al., Appl. Phys. Lett., 71, 656 (1997).

[15] R. K. Goodall, R. J. Jiggins, and J. P. Harrang, Phys. Rev. B 31, 6597 (1985); A. M. C. Valkering et. al., Phy. Rev. Lett. 81, 5398 (1998). 
[15] R. K. Goodall, R. J. Jiggins, and J. P. Harrang, Phys. Rev. B 31, 6597 (1985); A. M. C. Valkering et. al., Phy. Rev. Lett. 81, 5398 (1998).

[16] M. Büttiker, Phys. Rev. Lett. 57, 1761 (1986).

[17] A. M. Chang and J. E. Cunningham, Solid State Commun. 72, 651 (1989); L. P. Kouwenhoven et al., Phys. Rev. Lett. 64, 685 (1990).

[18] R. Morf and N. d'Ambrumenil, Phys. Rev. Lett. 74, 5116 (1995). 


\section{Figure Captions}

FIG. 1. Sample EA100 multi-terminal capacitance elements for (a) $\mathrm{C}_{\mathrm{G}, 1}$, (b) $\mathrm{C}_{\mathrm{G}, 3,}$, and (c)

$\mathrm{C}_{\mathrm{G}, 2}$. (d) Two-terminal capacitance $\mathrm{C}_{2 \mathrm{~T}}$, and simultaneously measured $\rho_{\mathrm{xx}}$. Inset shows sample geometry. Arrows indicate propagation direction of edge states for $\mathrm{B}>0$.

FIG. 2. The four-terminal resistance measured across the gate region vs. gate voltage at $\mathrm{T}=0.3 \mathrm{~K}$ from sample EA100. See the inset for the sample configuration. The labels denote $\left(v_{b}, v_{g}\right)$, the filling factors in the bulk and the gated regions.

FIG. 3. Sample EMC715 multi-terminal capacitance elements (a) $\mathrm{C}_{\mathrm{G}, 2}$ and (b) $\mathrm{C}_{\mathrm{G}, 1}$ and $\mathrm{C}_{\mathrm{G}, 3}$, near $v=3 / 2$. An anomalous capacitance peak centered at $v=3 / 2$ appears in $C_{G, 2}$. (c) Same as (a), but for several different temperatures, with curves vertically offset for clarity. 
Table 1. Sample parameters.

\begin{tabular}{l|c|c}
\hline Sample & $n\left(10^{11} \mathrm{~cm}^{-2}\right)$ & $\mu\left(\mathrm{cm}^{2} / \mathrm{Vs}\right)$ \\
\hline EMC715 (dark) & 2.6 & $1.1 \times 10^{6}$ \\
EA65 (dark) & 1.2 & $1.3 \times 10^{6}$ \\
EA100 (dark) & 1.3 & $1.2 \times 10^{6}$ \\
EA100 (LED) & 2.1 & $3.0 \times 10^{6}$
\end{tabular}



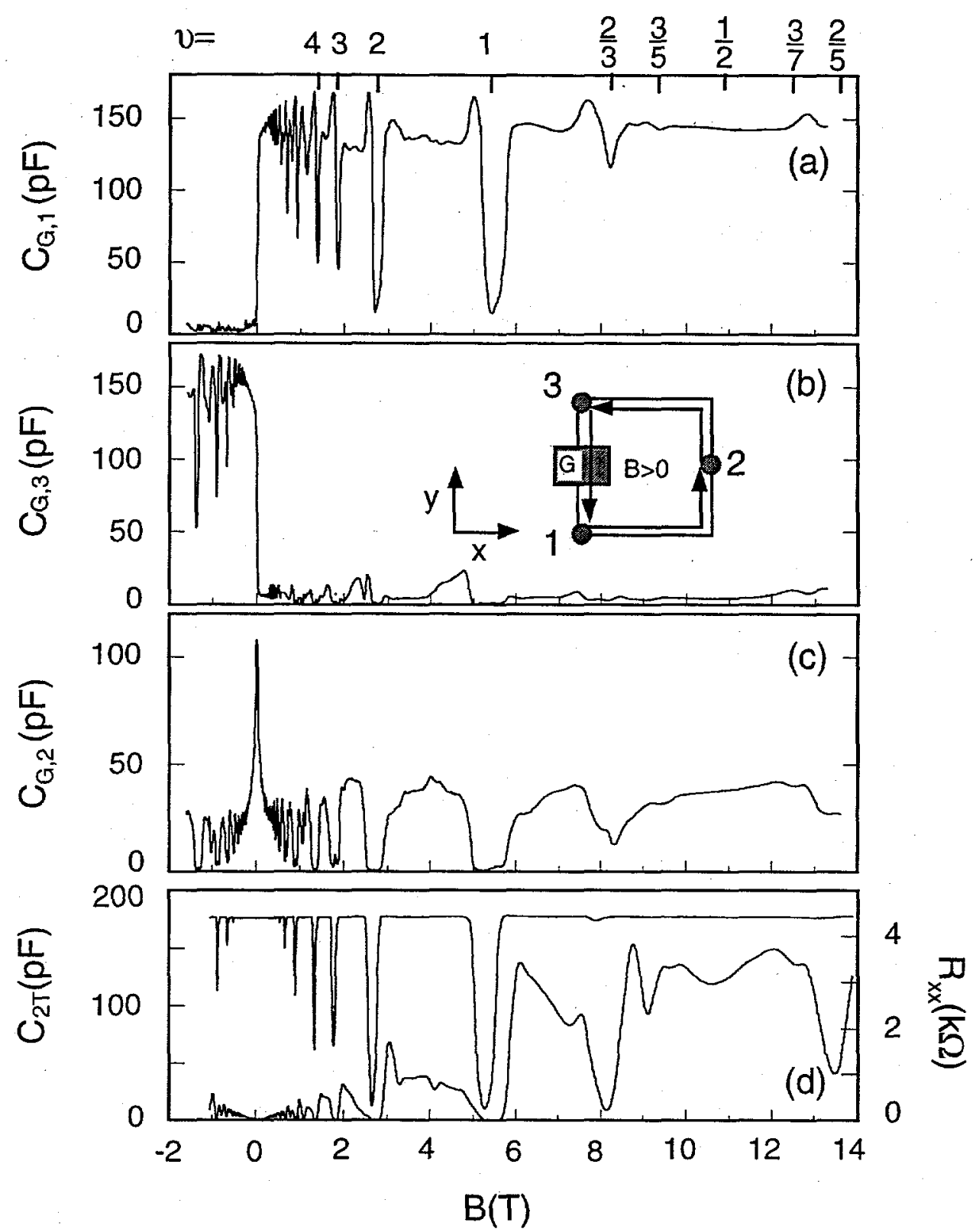

Figure 1

Moon et al. 


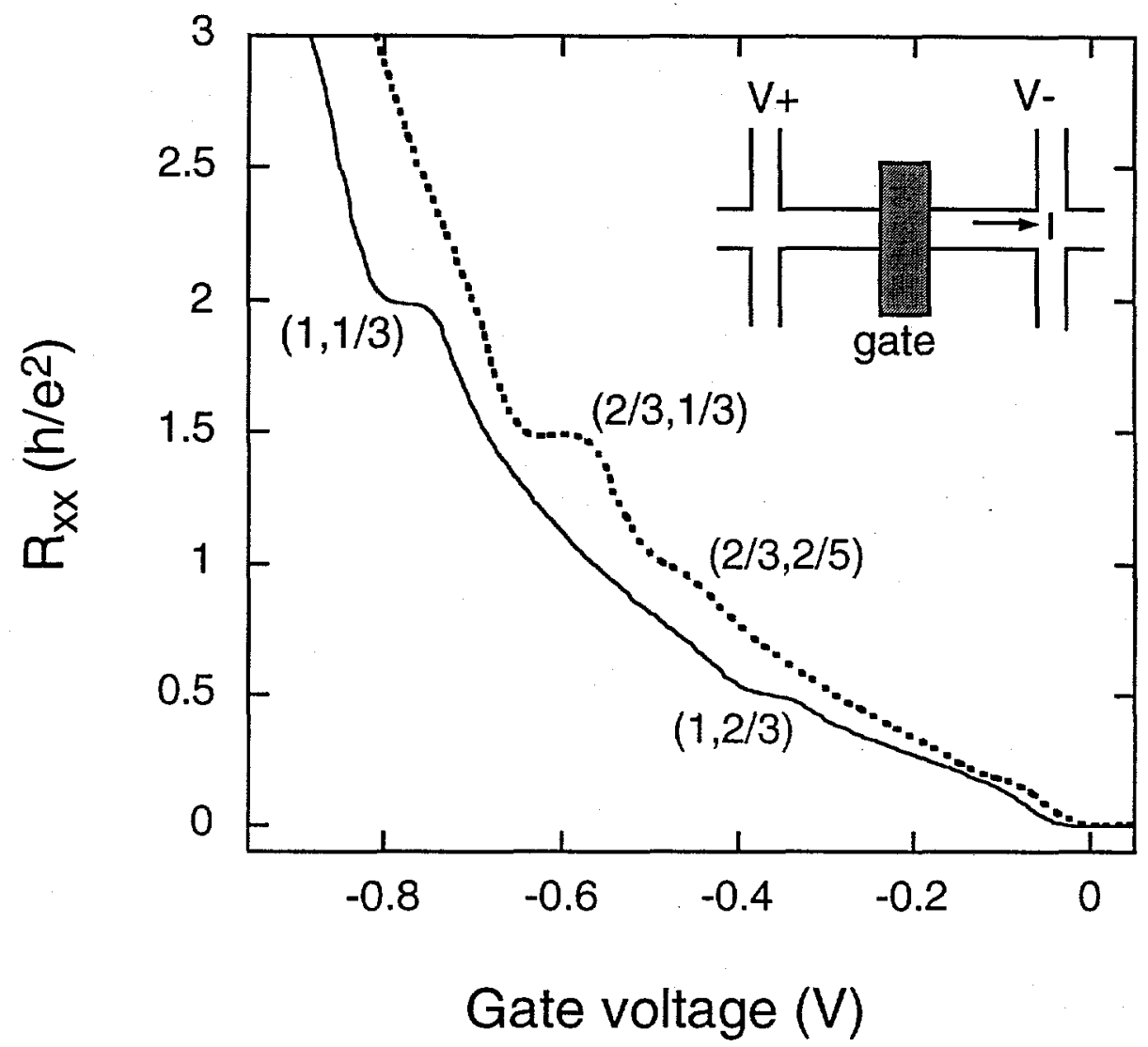

Figure. 2

Moon et al. 


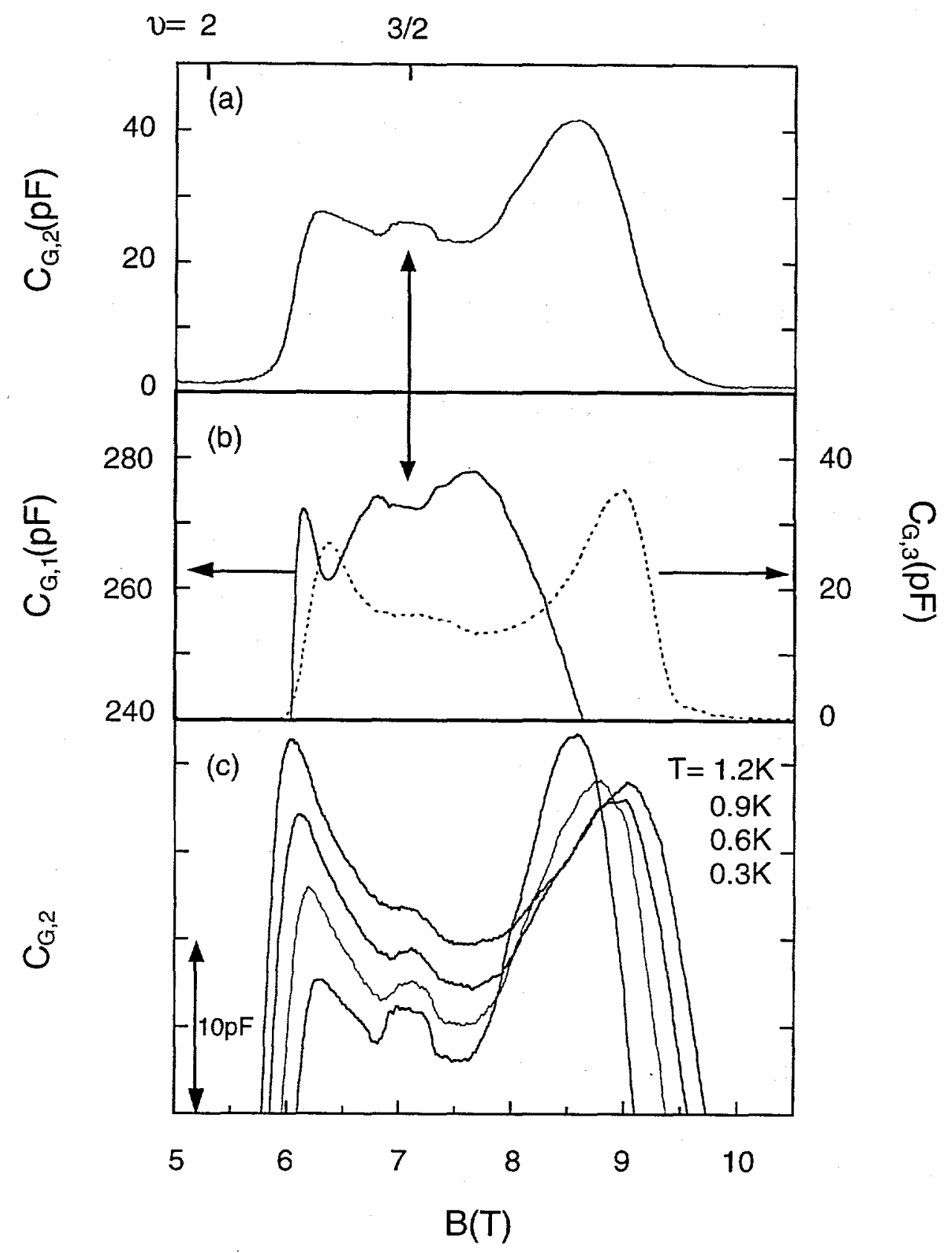

Figure 3 .

Moon et al. 